\title{
Public Health Risk from the Use of Phenylarsonic Feed Additives in China
}

\author{
YuANAN HU ${ }^{1}$, HeFA CHENG ${ }^{2}$, SHU TAO ${ }^{3}$, JERALD L. \\ $\mathrm{SCHNOOR}^{4}$ \\ ${ }^{1}$ School of Water Resources and Environment, China \\ University of Geosciences (Beijing), yhu@cugb.edu.cn \\ ${ }^{2}$ College of Urban and Environmental Sciences, Peking \\ University, hefac@pku.edu.cn \\ ${ }^{3}$ College of Urban and Environmental Sciences, Peking \\ University, taos@pku.edu.cn \\ ${ }^{4}$ Department of Civil and Environmental Engineering, \\ University of Iowa, jerald-schnoor@uiowa.edu
}

Phenylarsonic feed additives were once widely used in poultry and swine production around the world, which brought significant, and unnecessary, health risk to consumers due to elevated residues of arsenic species in animal tissues. To assess the public health risk of arsenic residues in chicken tissues, a survey on the content and speciation of arsenic in chicken tissues from live poultry markets was conducted in Guangdong province, China, between 2013 and 2014. The geometric mean content of total arsenic in the chicken meats from urban markets was found to be 1.7 times higher than that in the products from rural markets, and inorganic arsenic (i-As) accounts for approximately $30 \%$ of the total arsenic content in the chicken tissues. Based on the average contents of $i$-As in the chicken meats from local markets, lifetime consumption would result in 141 and 46 additional cases of lung and bladder cancer per million adults living in the urban and rural areas of Guangdong province, respectively. Although the chicken consumption rates of urban and rural populations in Guangdong were only 60 and $30 \%$ of that of the U.S. population, their incremental lifetime cancer risk (ILCR) from $i$-As exposure through chicken consumption was approximately 7.6 and 2.5 times higher, respectively, due to the use of phenylarsonic feed additives in chicken production at the time.

Out of concern for the negative impacts on human and ecosystem health, China banned the use of phenylarsonic feed additives on food animals on May 1, 2019. This ban, if fully enforced, would result in reduction of approximately 1,160 cancer cases per year from the consumption of chicken meat alone, and avoid an annual economic loss of nearly 0.6 billion CNY. This ban is also expected to bring even greater public health benefits due to the reduction in the accumulation of arsenic in food crops grown on the farmlands fertilized by poultry and swine wastes. 